\title{
Relating Experimental and Theoretical Perspectives in Nanomechanics
}

Vikas Tomar

With significant advances made in multiscale models and multiscale experiments, a significant gap exists between the two fields. Separately, both fields have shown a significant promise of discovery. Simulation can provide a cost effective predictability component. On the other hand, experiments can provide a validation and realization component. A significant effort is required to advance a synergistic effort between the two separate fields so that a strong unified validation and verification framework that advances discovery and novelty in different scientific fields can be established. With this point of view, articles in this collection focus on presenting advances made in five different fields where efforts focusing on synergistic advancements between simulations and experiments are important. The first article by A.V. Semichaevsky et al. (University of Illinois) focuses on low-dimensional semiconductor (LDS) structures such as quantum dots (QDs), quantum wires, and quantum wells in which electron and hole wave functions are confined due to heterogeneous composition and often strongly affected by mismatch strain. An overview of computational and experimental methods for characterization of QD heterostructures is provided. Particularly, focus is on reviewing their concerted efforts to bring together computation and experiment in order to better explain optoelectronic and photovoltaic properties of LDS structures.

Creep is non-recoverable high temperature plastic deformation occurring at low load regimes, constant stress val- ues, and small strain rates. While mechanistic information regarding macroscale (centimeters and higher) creep deformation has received significant attention in the past few decades, the nanoscale (few nanometers to a few hundred nanometers) and microscale (few hundred nanometers to a few hundred micrometers) creep behavior has recently become important. The second article by Ming Gan and Vikas Tomar (Purdue University) presents an overview of the recent developments in modeling and experiments focused on analyzing nanoscale and microscale temperature dependent creep in materials. Special emphasis is on presenting the latest advances regarding high-temperature multiscale creep measurements in materials as well as model based analyses. The next article by Yang Lu and Jun Lou (Rice University) presents an overview of the latest advances in quantitative mechanical characterization of one-dimensional metallic nanostructures, especially metallic nanowires, with diameters ranging from hundreds of nanometers to only a few nanometers. Ultrahigh mechanical strength and size dependent mechanical behaviors are unambiguously demonstrated with the aid of recently developed highly sensitive measurement techniques. Such developments are expected to have a significant impact on nanomechanical characterization of various technologically important one-dimensional nanomaterials and the understanding of their underlying deformation mechanisms.

Polymer based nanocomposites are notorious for difficulty in character- izing their failure modes and scarcity of models to predict their failure. The next article by Alvaro J. Mendoza Jasso and co-workers at Purdue University presents an advancement in the form of a combination of models and experiments in accurately predicting fiber failure in a polymer composite material. A model is demonstrated that incorporates the effect of uncertainty in the microstructure of composite systems in the failure analysis. The model determines the range of failure angles in an open hole off-axis specimen and the uncertainty in the critical effective shear strain in the matrix phase at failure due to uncertainties in the fiber volume fraction. The last article by Vikas Prakash et al. (Clemson University) presents an in-situ mechanical characterization and testing device. The development of the presented in-situ test and characterization device brings to the scientific community a capability to perform test and characterization of mechanical properties of nanostructures, including biological materials, while undergoing high resolution nanoscale imaging. A better fundamental characterization of mechanical response of nanoscale structures is expected to lead to a better understanding of the novel phenomena at the nanoscale and the underlying physics involved in the design of high performance nano-engineered materials and more reliable devices.

Vikas Tomar is an associate professor at Purdue University and a guest editor to JOM from the Nanomechanical Materials Behavior Committee of the Materials Processing \& Manufacturing Division of TMS.

\section{Vikas Tomar is a TMS Member!}

To read more about him, turn to page 15. To join TMS, visit www.tms.org/Society/Membership.aspx. 